Aim of the study: Horseshoe kidney is one of the most common genitourinary system anomalies. The frequency of its occurrence in the whole population is about $0.25 \%$. In the available literature more than 200 cases of such tumourdefect coexistence have been described. Purpose of this study was to present the possibilities of using various methods of surgical treatment of tumors localized in the horseshoe kidney.

Material and methods: In the years 2004-2007 five patients were diagnosed and treated for tumours in horseshoe kidneys. Ultrasonography, computed tomography, urography and nuclear magnetic resonance were applied in the diagnosis. During the treatment both open operations and modern methods of treating tumours with a diameter less than $40 \mathrm{~mm}$ (nephron sparing) were used.

Results: Renal cell carcinoma (RCC) was confirmed in all the patients.

Conclusions: Our observations confirm the fact that RCC is the most often diagnosed tumour in horseshoe kidneys. The prognostic factors are the same as in the case of tumours of the kidneys without any abnormalities. The diagnosis is based on applying the modern methods of imaging and in the treatment it is possible to apply the modern, non-invasive methods.

Key words: horseshoe kidney tumour, nephron-sparing surgery, laparoscopy.

\section{Tumour in horseshoe kidney - different surgical treatment shown in five example cases}

\author{
Michał Tkocz, Maciej Kupajski
}

Urology Departments, Prof. E. Michalowski Memorial Hospital in Katowice, Poland

\section{Introduction}

Horseshoe kidney is one of the most common developmental disorders of the genitourinary system, which forms between the $4^{\text {th }}$ and $6^{\text {th }}$ week of fetal life. It occurs in approximately 1-4 per 1,000 births and more often afflicts men than women. The defect may be accompanied by other developmental disorders of the genitourinary system and other organs. In the world literature, approximately 200 cases of tumours developing in the kidneys affected by this defect have been described. The risk of developing cancer in the kidneys with a disturbed development is similar to the kidneys with a normal structure, but the risks of Wilms' tumour and cancer of the transitional epithelium increases 2-6 times. Renal cell carcinoma (RCC) is the most common neoplasm developing in horseshoe kidneys [1-4].

\section{Material and methods}

From 2004 to 2007, five patients with tumours in horseshoe kidneys were treated in the urology department of the Professor Michalowski Hospital in Katowice. The observations of the patients were carried out prospectively. The average age of the patients was 63.2 (47-85). The presence of the developmental lesion was confirmed in the diagnostics of tumour symptoms and arterial hypertension. The preoperative diagnostics were conducted using ultrasonography (USG), urography, computed tomography (CT), and in single cases, nuclear magnetic resonance (NMR). Check-up examinations were carried out approximately 10 months after the surgery (6-36 months), using USG, CT, and $\mathrm{X}$-ray of the chest.

\section{Results}

All the removed tumours were renal cell carcinomas, four in the second stage of the Fuhrman classification, and one in the third. Haematuria was the first symptom of the tumour in one patient, in two cases pain was the reason for starting the diagnostics, and in the other cases both the tumour and the renal dysfunction were discovered accidentally during the diagnostics of other disorders (arterial hypertension).

In the preoperative diagnostics, all the patients had USG, CT and urography performed and one patient was diagnosed by NMR.

In one patient, the left half of the horseshoe kidney with the tumour was removed by a median incision (xipho-pubic) after previous separation of the organ. Due to the coexistence of hydrocele of the gallbladder, cholecystectomy was also conducted. In another patient, after separation of the kidneys and the preparation of the kidney with the tumour, heminephrectomy was conducted from the posterolateral access. In this case, the diameter of the tumour was $8 \mathrm{~cm}$.

In three patients, the lumbar posterolateral incision was used. In two other patients, after preparation of the kidney, only the tumour was removed by wedge resection, sparing the organ (Figs. 1, 2). Wedge resection of the tumour 
was performed in temporal ischemia after closing the pedicle 'en bloc' with the tissues surrounding the vessels, which was aimed at reducing injury of the arterial vessels. After closing segmental vessels, if the renal collecting system was damaged, it was closed with a continuous stitch. The surface of the cavity after the tumour was coagulated with argon (ForceArgon, Valleylab argon beam coagulation). The continuity of the renal parenchyma was reconstituted by single stitches. The next patient had the tumour removed laparoscopically from the retroperitoneal access, using four ports. In this case, after the closure of the pedicle vessels of the kidney, the tumour was excised with a harmonic knife (Ultracision EthiconEndo Surgery INC Johnson \& Johnson USA), haemostatic material was introduced into the cavity (TachoComb Nycomed GmbH Austria), and then the continuity of the parenchyma was reconstructed by single stitches (Table 1, 2).

In all the operated patients, the presence of a thick parenchymal isthmus was found during the operation. In four patients, the pathology of vascularisation was confirmed.

The check-up examinations are performed as in patients operated on due to renal cancer in the kidneys with undisturbed development. They include basic laboratory tests, USG, $\mathrm{CT}$ and $\mathrm{X}$-ray of the chest. During the check-up examinations, all the patients were free of metastases. One patient with concomitant nephrolithiasis of the horseshoe kidney half opposite to the tumour was subjected to ESWL treatment (extracorporeal shock-wave lithotripsy).

The average tumour size was $45.8 \mathrm{~mm}$ (35-80 mm); three tumours were located in the lower pole of the kidney, one in the upper pole and in the central part of the kidney. In three patients, the lesion was situated in the left kidney and in two patients in the right one. The mean hospitalization time was 10.4 days (11-16 days).

\section{Discussion}

Horseshoe kidney is one of the most common developmental disorders of the kidneys. Its incidence is estimated at approximately $0.25 \%$ of the total population. In 1976, Buntly presented 111 cases of tumours which developed in horseshoe kidneys. In his review, tumours of renal cell carcinoma origin amounted to $50 \%$, while the percentage of TCC and Wilms' tumour was each $25 \%$. Similar data are presented in other review papers available in the literature.

The group of patients presented by us was uniform in terms of the histopathological nature of the tumour - in all

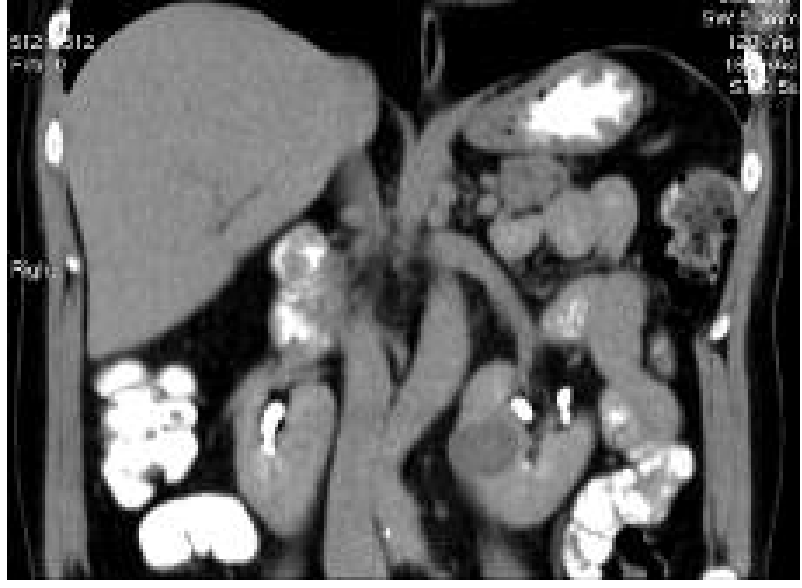

Fig. 1. CT - tumour in the left part of the horseshoe kidney before nephron sparing surgery (frontal section)

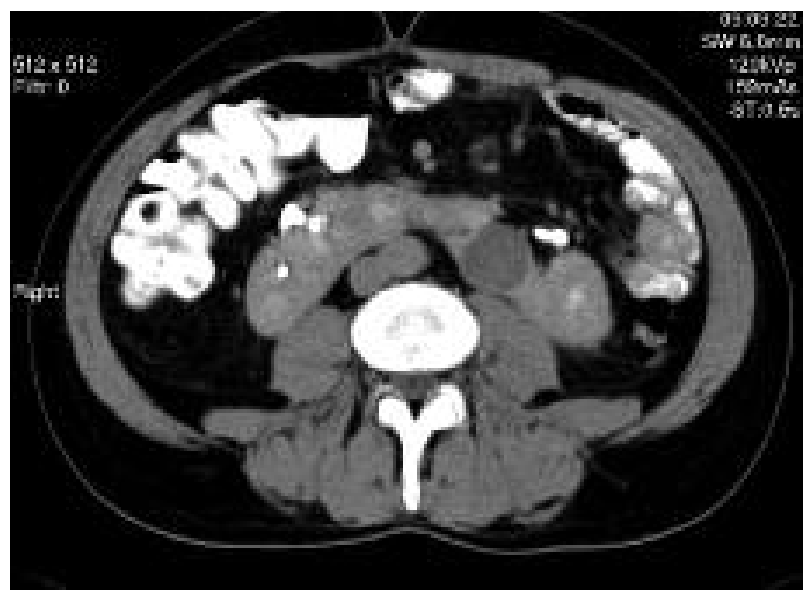

Fig. 2. CT - tumour in the left part of the horseshoe kidney - transverse section

of them the presence of RCC was confirmed. The incidence of RCC in horseshoe kidneys is the same as in normal kidneys, and its development and course are not related to the presence of the defect. According to the literature, transitional cell carcinoma (TCC) develops more frequently in horseshoe kidneys. The factors predisposing to its formation result from the presence of the defect: urinary obstruction, susceptibility to nephrolithiasis and chronic infection $[5,6]$.

As is clear from the data presented by the NWTSG (National Wilms Tumour Study Group), horseshoe kidneys occur in

Table 1. Characteristics of patients based on age, sex, clinical symptoms and diagnostic methods

\begin{tabular}{ccccc} 
No. & Age & Sex & Symptoms & haematuria \\
\hline 1 & 83 & $F$ & incidental & incidental \\
2 & 47 & $F$ & pain & USG, CT* L \\
\hline 3 & 72 & $M$ & pain
\end{tabular}

*stone in gallbladder coincidence

**nephrolithiasis of the left kidney coincidence 
Table 2. Characteristics of patients with regard to tumor location, surgical approach, type of surgical procedure, histological type and grading

\begin{tabular}{|c|c|c|c|c|c|}
\hline No. & Location & Operative access & Kind of treatment & HP & Grade \\
\hline 1 & middle part (37 mm) & xipho-pubic & heminephrectomy and cholecystectomy & $\mathrm{RCC} \mathrm{II}^{*}$ & pT1NoMo \\
\hline 2 & lower pole (80 mm) & lumbotomy & heminephrectomy & $\mathrm{RCC} \|$ & pT2NoMo \\
\hline 3 & upper pole $(40$ mm) & laparoscopy & nephron sparing surgery (tumour excision) & RCC III & pT1NoMx \\
\hline 4 & lower pole (35 mm) & lumbotomy & nephron sparing surgery (tumour excision) & RCC II & pT1NoMo \\
\hline 5 & upper pole (37 mm) & lumbotomy & nephron sparing surgery (tumour excision) & RCC II & pT1NoMo \\
\hline
\end{tabular}

$0.49 \%$ of patients with confirmed Wilms' tumour. According to Neville et al., a relationship between the development of Wilms' tumour and the chromosomal deletion 11p13 cannot be excluded. This should lead to genetic testing in children afflicted by this defect $[4,7]$.

In the diagnostics of neoplasms in horseshoe kidney, the same imaging methods as in kidneys with an undisturbed development are used. In the group presented by us, the basic examination was USG. However, in order to fully assess the nature of the lesion, we performed CT complemented by NMR in individual cases $[8,9]$.

The surgical treatment of horseshoe kidney cancer is based on the same principles as treatment of kidneys whose development was undisturbed. These guidelines are a continuation of the universal principles of the radical nephrectomy implemented by Robson in 1963, but undergoing evolution especially for the so-called small tumours with a diameter not exceeding $4 \mathrm{~cm}$. A partial nephrectomy was first proposed by Wells in 1884, but only as late as in 1887 did Czerny perform a partial resection of the renal parenchyma due to a malignant lesion (quote after 9). In 1950, Vermooten identified possibilities and technical aspects of removing the tumour while sparing the organ. In three patients from the presented group, we used the indication of choice for tumours with a diameter not exceeding $40 \mathrm{~mm}$, with a favourable location which enables their removal while maintaining a safe surgical margin.

In the analysed group of patients, heminephrectomy was performed in two cases. In the first one, it was due to the location of the tumour in the central part of the kidney, age of the operated patient ( $>80$ years old) and the coexistence of hydrocele of the gallbladder (case no. 1), while in the latter one, the size of the tumour $(8 \mathrm{~cm})$ was the reason for choosing such a procedure. In other cases, we decided to use the method of choice which spares the organ - two patients had wedge resection of the tumour performed by means of open surgery, while in one patient we conducted resection of the pole with the tumour by retroperitoneal laparoscopy. In this case, the tumour was located in the upper pole, it expanded outside of the kidney, and its size did not exceed $40 \mathrm{~mm}$. In two other cases, the tumours were located in the lower part of the kidney, they did not reach the isthmus, and their diameter was smaller than $40 \mathrm{~mm}$. When making a decision to use methods which spare the organ in horseshoe kidneys, three main factors resulting from the essence of the defect should be borne in mind: variability in the course of blood vessels (presence of straying vessels), different structures of the isth- mus, and anatomical location of the organ. A single renal artery is found only in about $30 \%$ of horseshoe kidney cases. In other cases, numerous anomalies of the vasculature of the kidney and the isthmuses are possible. The isthmus, depending on its structure (parenchymal or fibrous), is supplied by numerous straying vessels. Such large variation in vasculature may restrict the use of methods which enable sparing of the organ.

In 2001, Lee, Hilton and Russo from the Memorial SloanKettering Cancer Center proposed the use of three-dimensional spiral computed tomography to accurately localize the tumour in horseshoe kidney in relation to the renal collecting system and the vascular tree, which enables one to take a decision about organ-conserving surgery. The literature described single cases of preoperative supraselective embolization of the vessels supplying the tumour, which makes its removal by means of minimally invasive procedures possible and safe [10, 11].

Tumours large in size, with an unfavourable localization within the horseshoe kidney, unclear location of the tumour in relation to the vessels and the renal collecting system, and a thick isthmus with the system of straying vessels should, in our opinion, suggest the operator to remove the tumour through open surgery from the intraperitoneal access or in selected cases from the lumbar posterolateral access [12].

In the group of patients presented by the authors, the surgical access and the method of treatment were selected individually for each patient considering their anatomical differences resulting from the essence of the defect, tumour size, and concomitant ailments. In the case of kidneys with a thin fibrous isthmus and a well-defined location of the tumour, a decision to use the lumbar posterolateral access in three patients was made. The use of laparoscopy resulted from the location of the tumour and its size, while the choice of the intraperitoneal access was a consequence of the coexistence of the large gallbladder hydrocele and the general condition of the patient.

\section{Conclusions}

According to the literature and our observations, RCC is the most common neoplasm found in kidneys of disturbed development. However, its incidence is not greater than in the general population, and prognostic factors are the same as in kidneys of undisturbed development. In patients with Wilms' tumour, the incidence of this tumour in horseshoe kidneys is approximately $0.48 \%$.

The diagnosis of neoplasms in horseshoe kidneys is based on the same imaging methods as in normal kidneys, but it 
appears that spiral CT and magnetic resonance enable such imaging of the lesions that modern, minimally invasive methods of surgical treatment can be used.

Using the organ-conserving method in both open surgery and laparoscopy can be an attractive alternative procedure for tumours which satisfy the criteria of a partial excision of choice in horseshoe kidneys.

\section{References}

1. Bauer SB. Anomalies of the upper urinary tract. In: Wein AJ, Kavoussi LR, Novick AC, Partin AW, Peters CA. Campbell-Walsh Urology. Ed. 9. Saunders, Philadelphia 2007; 3269-3334.

2. Buntley D. Malignancy associated with horseshoe kidney. Urology 1976; 8: 146-148.

3. Harris J, Robert E, Källén B. Epidemiologic characteristics of kidney malformations. Eur J Epidemiol 2000; 16: 985-992.

4. Stimac G, Dimanovski J, Ruzic B, Spajic B, Kraus O. Tumors and kidney fusion anomalies - report of five case and review of the liter ature. Scand J Urol Nephrol 2004; 38: 485-489.

5. Hohenfellner M, Schultz-Lampel D, Lampel A, Steinbach F, Cramer BM, Thüroff JW. Tumor in horseshoe kidney: clinical implications and review of embryogenesis. J Urol 1992; 147: 1098-1102.

6. Rubio Briones J, Regalado Pareja R, Sánchez Martín F, Chéchile Toniolo G, Huguet Pérez J, Villavicencio Mavrich H. Incidence of tumoural pathology horseshoe kidneys. Eur Urol 1998; 33: 175-179.

7. Neville H, Ritchey ML, Shamberger RC, Haase G, Perlman S, Yoshioka T. The occurrence of Wilms tumor in horseshoe kidneys: a report from National Wilms Tumor Study Group (NWTSG). J Pediatr Surg 2002; 37: 1134-1137.

8. Robson CJ. Radical nephrectomy for renal cell carcinoma. J Urol 1963; 89: 37-42.

9. Uzzo RG, Novick AC. Nephron sparing surgery for renal tumors: indications, techniques and outcomes. J Urol 2001; 166: 6-18.

10. Yoshinaga K, Kodama K, Tanii I, Toshimori K. Morphological study of a horseshoe kidney with special reference to the vascular system. Anat Sci Int 2002; 77: 134-139.

11. Lee CT, Hilton S, Russo P. Renal mass within a horseshoe kidney: preoperative evaluation with three-dimensional helical computed tomography. Urology 2001; 57: 168-170.

12. Kim TH. Renal cell carcinoma in a horseshoe kidney and preoperative superselective renal artery embolization: a case report. Korean I Radiol 2005; 6: 200-203.

\section{Address for correspondence}

Michat Tkocz MD

Urology Department

Prof. E. Michalowski Memorial Hospita

Wietnamska $59 \mathrm{~K}$

40-765 Katowice, Poland

e-mail: tkocz40@interia.pl

Submitted: $\quad 1.10 .2009$

Accepted: $\quad 29.11 .2011$ 